\title{
A CRISE DE PARADIGMAS NA CIÊNCIA E AS NOVAS PERSPECTIVAS PARA A ENFERMAGEM
}

\author{
The crisis of paradigms in science and the new perspectives in nursing \\ La crisis de paradigmas en la ciencia y las nuevas perspectivas en enfermería
}

Quintila Garcia Santos

Dulcian Medeiros de Azevedo²

Roberta Kaliny de Sousa Costa ${ }^{3}$

Flávio Pereira de Medeiros ${ }^{4}$

\section{RESUMO}

0 paradigma é entendido como um padrão a ser seguido, e inclui o conjunto de crenças, valores, técnicas e teorias partilhadas, sendo influenciado por fatores culturais, políticos, econômicos e sociais vigentes. Objetivou-se realizar uma reflexão acerca da crise paradigmática do conhecimento científico e de suas implicações no âmbito da enfermagem, apresentando uma visão panorâmica do desenvolvimento da pesquisa nessa área. É premente a necessidade de novos princípios nor teadores da prática científica, os quais vêm emergindo na ética, sustentabilidade ambiental, interdisciplinaridade, valorização da complexidade, respeito à subjetividade. Para que a enfermagem se fortaleça enquanto ciência, profissão e prática social, é imprescindível a utilização das ferramentas disponíveis, na promoção de ações sociopolíticas que apontem para uma maior qualidade de vida da sociedade. 0 processo do trabalho em saúde e seus paradigmas exigem do enfermeiro clareza dos seus limites de atuação, intervenção e habilidade para construir conhecimento novo, promovendo a inovação da produção em saúde.

Palavras-chave: Enfermagem. Pesquisa em enfermagem. Filosofia em enfermagem.

\begin{abstract}
The paradigm is understood as a pattern to be followed, and includes the set of beliefs, values, techniques and shared theories and is influenced by cultural, political, economic and social laws. The objective was to conduct an analysis about the paradigmatic crisis of scientific knowledge and its implications in nursing, providing an overview of the development of a research in this area. The need for new guiding principles of scientific practice is urgently needed, which are emerging in ethics, environmental sustainability, interdisciplinary, appreciation of complexity, and respect for subjectivity. For nursing to become stronger as a science, profession and social practice is essential to use the tools available in promoting socio-political actions that point to a higher quality of life of society. The work process in health and its paradigms require from the nurse clarity of its limited scope, intervention, and ability to build new knowledge, promoting innovation in the health production.
\end{abstract}

Keywords: Nursing. Nursing Research. Philosophy. Nursing.

\section{Resumen}

Se entiende el paradigma como un patrón a seguir, e incluye el conjunto de creencias, valores, técnicas y teorías para compartir, y está influenciado por factores culturales, políticos, económicos y sociales vigentes. El objetivo fue realizar un análisis sobre la crisis paradigmática del conocimiento científico y sus implicaciones en la enfermería, presentando un panorama general del desarrollo de la investigación en este ámbito. Se necesita con urgencia de nuevos principios que guíen la práctica científica que están surgiendo en la ética, la sostenibilidad ambiental, la interdisciplinariedad, la apreciación de la complejidad, y el respeto de la subjetividad. Para fortalecer la enfermería como una ciencia, profesión y la práctica social es esencial utilizar las herramientas disponibles en la promoción de acciones sociopolíticas que apuntan a una mejor calidad de vida de la sociedad. El proceso de trabajo en salud y sus paradigmas requieren percepción de los enfermeros de sus límites de actuación, la intervención y la capacidad para construir nuevos conocimientos, promoviendo la innovación en la producción de salud.

Palabras-Claves: Enfermería. Investigación en Enfermería. Filosofía en Enfermería.

\footnotetext{
${ }^{1}$ Discente do Curso de Graduação em Enfermagem (6º Período), Universidade do Estado do Rio Grande do Norte (UERN), Campus Caicó. Caicó-RN. Brasil. E-mail: quintilagarcia@yahoo.com.br; ²Enfermeiro/Docente. Curso de Graduação em Enfermagem (UERN), Campus Caicó, Caicó-RN. Mestre em Enfermagem (PGENF/UFRN) e Doutorando em Ciências da Saúde (PPGCSa/UFRN). Líder do Grupo de Pesquisa "A enfermagem no processo saúde-doença individual/ coletiva, na educação em saúde e na assistência/gerência de serviços de saúde". Caicó-RN. Brasil. E-mail: professordulcian@gmail.com; ${ }^{2}$ Enfermeira/ Docente. Curso de Graduação em Enfermagem (UERN), Campus Caicó, Caicó-RN. Mestre em Enfermagem (PGENF/UFRN) e Doutoranda em Ciências da Saúde (PPGCSa/UFRN). Vice-Líder do Grupo de Pesquisa "A enfermagem no processo saúde-doença individual/coletiva, na educação em saúde e na assistência/gerência de serviços de saúde". Caicó-RN. Brasil. E-mail: robertaksc@bol.com.br; "Discente do Curso de Graduação em Enfermagem (6º Período - UERN), Campus Caicó, Caicó-RN. Brasil. E-mail: pikeno26@hotmail.com
} 


\section{INTRODUCÃO}

Apesar de a ciência ser considerada um mecanismo importante e eficiente para a busca e desenvolvimento do conhecimento, chama atenção a forte intervenção do homem na natureza em detrimento do equilíbrio necessário à conservação da vida. A humanidade, ao enveredar pela busca do desenvolvimento econômico desenfreado pautado pelo exercício do conhecimento científico, vem alterando a ordem natural de muitos fenômenos e arriscando a sobrevivência humana, de outras espécies e do planeta.

Novas formas de estudo emergem como complemento ao modelo científico positivista, propondo uma ciência pautada na valorização do homem e da biodiversidade, em busca de construir sociedades mais saudáveis em seus diversos aspectos.

A enfermagem, enquanto profissão, nasceu sob a influência do capitalismo, imprimindo uma intensa divisão do trabalho em saúde, com o conhecimento manifesto e produzido centrado na doença. Um dos seus desafios atuais é ressignificar seu processo de trabalho e estabelecer-se enquanto ciência comprometida com o social.

Este artigo foi produzido a partir da Disciplina Processo de Investigação em Enfermagem, do Curso de Graduação em Enfermagem, Universidade do Estado do Rio Grande do Norte (UERN), com a finalidade de realizar uma reflexão acerca da crise paradigmática do conhecimento científico e de suas implicações no âmbito da enfermagem, apresentando uma visão panorâmica do desenvolvimento da pesquisa nessa área.

\section{A CRISE PARADIGMÁTICA CONHECIMENTO CIENTÍFICO}

0 paradigma representa um padrão a ser seguido no âmbito científico ou social. Na comunidade científica, inclui crenças, valores, técnicas e teorias partilhadas, sendo influenciado pelos fatores culturais, políticos, econômicos e sociais vigentes. ${ }^{1,2}$ Portanto, a forma como um cientista vê um aspecto de seu mundo orienta o paradigma em que está trabalhando, e vice-versa, consubstanciando-se em critério de julgamento daquilo que é verdadeiro e real. ${ }^{1,2}$

Por reconhecer que um processo histórico não se dá linearmente ou de forma brusca, tendo em vista que a história é constituída por avanços, retrocessos, acontecimentos concomitantes, e ainda momentos transitórios, intenta-se que a descrição a seguir tenha uma visão macroscópica. Para tanto, serão trazidos à tona marcos históricos determinantes do paradigma científico e da ideia de ciência atual.

Enquanto paradigma dominante da ciência moderna, ${ }^{3}$ o positivismo teve suas bases no iluminismo, mas foi semeado ainda no Renascimento, quando ressurgiu a ideia da ciência racionalista da antiguidade. Nesse contexto, século XVI, Copérnico negou o geocentrismo e, em 1610, Galileu confirmou a Teoria Heliocêntrica, utilizando-se de conhecimentos matemáticos e geométricos. Em 1620, Bacon destacou o cunho utilitarista do conhecimento científico, apresentando um método de características práticas, o método indutivo. ${ }^{4}$

Em seguida, Decartes (1630) reafirmou a ideia do conhecimento científico originário de um rigoroso processo metodológico, enfatizando a ideia da produção do conhecimento a partir da razão. ${ }^{1,4}$ Com o anúncio deste modelo analítico, denominado posteriormente de reducionista, a busca pelo conhecimento passou ser orientada pelo estudo das partes que, ao serem unidas, fornecem uma visão do todo, modelo entendido nesse período como um mecanismo facilitador da produção do conhecimento. 0 método cartesiano tornou-se "sinônimo" de método científico a partir da Lei Universal da Gravidade de Isaac Newton (1700).

No início do século XIX, Augusto Comte estendeu esses preceitos às ciências sociais ao declarar que há uma ordem natural imutável, através da qual o cientista social deve se pautar na geração do conhecimento, alimentando a ideologia da neutralidade científica. Nasceu o positivismo, influenciado também pelos mecanismos de produção, a despeito da divisão e especialização no trabalho e, consequentemente, na ciência.

A partir do século XX, o paradigma dominante entra em crise $^{3}$, passando a ser questionado, sobretudo, a partir da Teoria da Relatividade de Albert Einstein e da Teoria Quântica de Max Planck, desmistificando a ideia do conhecimento científico indubitável. ${ }^{4}$

Aliado a tais acontecimentos, a transição paradigmática vivenciada também é oriunda da revolução científica iniciada pela física no século XX², e pela crítica à ciência no pós-guerra. Representa uma crise de verdades e de compreensão da realidade, que gera um descontentamento quanto às concepções e aos métodos existentes de observar o mundo e de fazer sentido nele, processo que dá origem a outras ideias e perspectivas. ${ }^{1}$

Nesse sentido, apesar de se reconhecer que o desenvolvimento científico trouxe inúmeros benefícios para a vida humana, percebe-se que sua prática desmedida e sem controle contribuiu para o agravo de problemas ambientais e desigualdades sociais, surgindo um modelo de sociedade individualista e desequilibrado. É premente a necessidade de novos princípios norteadores da prática científica, os quais vêm emergindo na ética, na sustentabilidade ambiental, na interdisciplinaridade, na valorização da complexidade, no respeito à subjetividade, à cultura e aos saberes alternativos, culminando na busca de relações mais saudáveis entre o homem e a natureza.

\section{PERSPECTIVAS EMERGENTES DA CRISE NA PESQUISA EM ENFERMAGEM}

Resguardando-se as devidas proporções dos acontecimentos históricos, pretende-se aqui resgatar, de maneira breve, a história da enfermagem, o surgimento de parâmetros científicos para a profissão e o desenvolvimento da pesquisa na área. 
A enfermagem, enquanto construção histórica, teve suas raízes a partir de práticas empíricas, em que as mulheres cuidavam dos doentes da família e cujos conhecimentos provinham do senso comum, do aprendizado recebido de seus ancestrais e da intuição. Na Idade Média, a enfermagem passou a ser direcionada aos pobres e exercida nas casas de misericórdia por membros da Igreja Católica, que viam a doença como uma questão espiritual e cuidavam dos doentes, vislumbrando a salvação de suas próprias almas. ${ }^{5}$

Com a superação do paradigma medieval e a ascensão da indústria, ultrapassou-se essa perspectiva prática de atuação. Junto com a industrialização e com as necessidades dela provenientes, emergiu o modelo hospitalar no século XVIII, com total influência do saber e prática médica na enfermagem, sendo essa, nesse momento, apenas um instrumento do trabalho daquela. ${ }^{5}$

No século XIX, tais relações tornaram-se ainda mais hierarquizadas, quando Florence Nightingale organizou a enfermagem moderna e subdividiu-a hierarquicamente, reproduzindo o modelo de sociedade classista (capitalismo), e o parâmetro científico da época (positivista). A partir dela, teve início também o processo investigativo na enfermagem, que, embora tenha apontado para um entendimento mais abrangente acerca do processo saúde-doença, nasceu pautado no modelo cartesiano., ${ }^{4,6}$

Ao chegar à América do Norte, o modelo nightingaleano ocasionou mudanças no ensino, de forma que a enfermagem norte-americana deu seus primeiros passos na investigação científica. No início do século XX, ocupou-se com pesquisas quantitativas, objetivando definir e quantificar os profissionais da enfermagem. Em seguida, converteu-se no fazer das enfermeiras, retomando às técnicas utilizadas no cuidado. ${ }^{6}$

Dessa forma, os princípios científicos adotados pela enfermagem até a segunda metade do século estiveram, predominantemente, ligados ao biológico. A partir de 1950, as pesquisas se voltaram para fenômenos de contextualização cultural, social e econômica, com grande repercussão no processo saúde-doença, destacando-se o conhecimento a partir das teorias de enfermagem.

Ao mesmo tempo em que passou a se reconhecer como uma prática social, a enfermagem começou sua busca pela compreensão das relações socioeconômicas, dentro de uma dialética social e histórica, em que as questões de poder influenciam diretamente a qualidade de vida e, consequentemente, a saúde da população. Nesse contexto, surgiram novas perspectivas para a pesquisa em enfermagem, adotando-se desenhos qualitativos.

No Brasil, a pesquisa em enfermagem seguiu uma linha semelhante à adotada pela enfermagem norte-americana, dada pela inserção de enfermeiras vindas dos Estados Unidos para auxiliarem no combate às epidemias que arrasavam o Brasil no início do século $X X^{6,7} \mathrm{e}$, anos mais tarde, por suas influências na formação/ensino de enfermagem.
Com a implantação das Escolas de Enfermagem, nível técnico e superior, e o surgimento de um elemento de divulgação científica em 1932, o "Annaes de Enfermagem", a pesquisa científica deu os primeiros passos no Brasil. As publicações feitas até a década de 1970 relacionavam-se, sobretudo, ao aspecto técnico, quantificável.

No contexto da reforma universitária de 1968, a pesquisa passou a ser difundida, atrelada ao ensino nas universidades. ${ }^{6}$ Foi a partir da reforma que os cursos de pósgraduação em enfermagem (nível mestrado) começaram a surgir, o primeiro em 1972, incrementando ainda mais a pesquisa no âmbito da enfermagem ao estreitar o vínculo entre a pós graduação e o desenvolvimento da pesquisa no Brasil. ${ }^{8}$

A partir da década de 1980, os cursos de doutorado em enfermagem foram abertos, e, após a ditadura, introduziramse na pesquisa deste nível reflexões sociais e filosóficas acerca das contradições existentes na sociedade e suas relações com a saúde. A enfermagem brasileira continuou buscando, com influência norte-americana, afirmar-se como ciência. ${ }^{6,8}$

$\mathrm{Na}$ atualidade, a produção científica no âmbito da enfermagem brasileira é de caráter predominantemente acadêmico, recebendo influências de paradigmas emergentes, ${ }^{9-}$ ${ }^{11}$ com mudanças significativas no ensino, pesquisa e extensão, almejando a interdisciplinaridade e a transformação do modelo assistencial em saúde.

Para que a Enfermagem se fortaleça enquanto ciência, profissão e prática social, é imprescindível a utilização das ferramentas disponíveis (pesquisa e extensão universitárias, controle social, parceria público-privada, trabalho voluntário) para promover ações sociopolíticas que apontem para uma maior qualidade de vida da sociedade. Esse tipo de proposta já é visível na prática, como verificado na pesquisa convergente assistencial, associando-se assistência e pesquisa, com o intuito de construir mudanças e/ou inovações na prática. ${ }^{12}$

Dessa forma, o desafio não seria somente trazer novas perspectivas para a pesquisa, dada a realidade da crescente produção atual, mas sim fazer com que o conhecimento produzido chegue à realidade dos serviços de saúde, do trabalho em comunidade, dos centros de formação, acarretando mudanças significativas, não se restringindo ao universo acadêmico.

O Sistema Único de Saúde (SUS), tal como se encontra estruturado, está inserido em um contexto político-econômicosocial de crise paradigmática na saúde, desencadeada pela também crise estrutural do capitalismo, que caracterizou uma nova etapa da economia mundial. Tal crise é dominada pela abertura de fronteiras econômicas intercontinentais, pelos avanços tecnológicos e pela redução da intervenção estatal, ordenada pelos ideais da globalização e do neoliberalismo econômico, provocando mudanças na estrutura organizacional de todo o sistema, para que um novo modelo possa ser construído. 
Atualmente, as iniciativas de mudança neste setor de assistência à saúde tem encontrado na Estratégia Saúde da Família (ESF) uma alternativa para substituição do modelo hegemônico, individualizado e biologicista. Orienta-se pela atenção voltada às ações de vigilância da saúde, através do trabalho em equipe com atividades assistenciais direcionadas para a saúde da coletividade, e pela concepção de saúde centrada no desenvolvimento de ações intersetoriais e interdisciplinares.

Entretanto, a operacionalização dessa estratégia sugere transformações profundas nas relações entre os usuários e profissionais, e destes entre si, para que as mudanças envolvendo o cuidado em saúde também possam ocorrer. Isso remete ao surgimento de novos paradigmas abrangendo a educação e o desenvolvimento dos recursos humanos em saúde, em especial os profissionais que compõem a ESF.

Nessa perspectiva, as Diretrizes Curriculares Nacionais orientam a busca dos centros de formação pela ressignificação do processo de formação em saúde, valorizando a interdisciplinaridade e a transversalidade dos conteúdos, e a incorporação de valores e discussões antropológicas, sociais e filosóficas, na busca de articulação entre teoria e prática.

\section{DA CRISE DE PARADIGMAS À ENFERMAGEM HOJ E}

0 processo do trabalho em saúde e seus paradigmas exigem do enfermeiro clareza dos seus limites de atuação, intervenção e habilidade para construir conhecimento novo, que promova a inovação da produção em saúde, atendendo às necessidades do serviço, da comunidade e do fortalecimento da própria categoria profissional, enquanto prática social historicamente constituída.

Tal situação subjaz a reflexão sobre as transformações paradigmáticas na enfermagem, sugerindo aos profissionais a ampliação do seu campo de visão/ atuação para qualificar a produção de saberes e o modo de agir em saúde, como forma de relativizar os valores do paradigma biologicista, tido como dominante.

Os novos paradigmas (teoria dos sistemas, interdisciplinaridade, complexidade, valorização da subjetividade, entre outros), configuram-se como uma esperança de que as pesquisas e o trabalho no âmbito da saúde passem a ter mais eficácia a partir de uma visão contextualizada e do respeito às formas diversas de conhecimento. Não se trata, por conseguinte, de negar a contribuição do positivismo para a ciência moderna, mas de reconstruir determinados conhecimentos que não mais satisfazem às demandas sociais, e se coadunem com propostas pautadas em uma ética de preservação e de construção de relações mais saudáveis entre o homem e a natureza.
Nesse sentido, a enfermagem passa a valorizar a unicidade da existência humana, a humanização da assistência e um cuidado que considera o indivíduo e seus aspectos bio-psico-sociais, inserido em um contexto social e comunitário. Esta tendência expressa a mudança da visão da profissão e reforça a ideia de que os novos significados da sua prática advêm de uma crise maior, uma importante mudança paradigmática (ou visão de mundo) que permeia o conhecimento científico produzido e afeta o caráter da educação, da prática e da pesquisa.

\section{REFERÊNCIAS}

1. Azevedo DM, Costa LM, Almeida Júnior JJ, Enders BC, Menezes RMP. Paradigmas emergentes: um ensaio analítico. Rev Eletr Enferm [periódico on-line] 2008; [citado 2010 jan 12 jan]; 10(3):835-42 Disponível em: http://www.fen.ufg.br/revista/v10/n3/pdf/ v10n3a30.pdf

2. Kuhn T. A estrutura das revoluções científicas. São Paulo: Perspectiva; 1970.

3. Santos BS. Um discurso sobre as ciências. $3^{\mathrm{a}}$ ed. São Paulo: Cortez; 2005.

4. Pereira WR, Bellato R. A crise de paradigma e a enfermagem. Texto\&Contexto Enferm. 1997 set/dez; 6(3): 113-30.

5. Almeida MCP, Rocha JSY. Primeiras expressões do saber de enfermagem. In:___ . 0 saber de enfermagem e sua dimensão prática. $2^{\mathrm{a}}$ ed. São Paulo: Cortez; 1989. p. 29-67.

6. Lopes GT. A trajetória da investigação científica no âmbito da enfermagem. Esc Anna Nery. 2002 abr; 6(1): 53-62.

7. Almeida AM, Oliveira ERA, Garcia TR. Pesquisa em enfermagem e 0 positivismo. Rev Esc Enferm USP. 1996 abr; 30(1): 25-32.

8. Santos TCF, Gomes MLB. Nexos entre pós-graduação e pesquisa em enfermagem no Brasil. Rev Bras Enferm. 2007 jan/fev; 60(16): 91-5.

9. Monteiro EMLM, Rolim KMC, Machado MFAS, Moreira RVO. A visão ecológica: uma teia na enfermagem. Rev Bras Enferm. 2005 maio/ jun; 58(3): 341-44.

10. Tier CG, Lundardi VL, Santos SSC. Cuidado ao idoso deprimido e institucionalizado à luz da complexidade. Rev Eletr Enferm. [periódico on-line] 2008; [citado 2010 jan 12]. 10(2): 530-36. Disponível em: http://www.fen.ufg.br/revista/v10/n2/pdf/v10n2a24.pdf 
11. Carvalho V. Por uma epistemologia do cuidado de enfermagem e a formação dos sujeitos do conhecimento na área da enfermagem:do ângulo de uma visão filosófica. Esc Anna Nery. 2009 abr/jun; 13(2): 406-14.

12. Paim L, Trentini M, Madureira VSF, Stamm M. Pesquisa convergenteassistencial e sua aplicação em cenários da enfermagem. Cogitare Enferm. 2008 jul/set; 13(3): 380-86. 